\title{
Workplace-based prevention and rehabilitation programs in Swedish public human service organisations
}

\author{
Stig Vinberg \\ Department of Health Sciences, Mid Sweden University, Sweden. stig.vinberg@miun.se
}

Background: This presentation focuses on results from studies concerning workplace health and rehabilitation interventions in one Swedish rural municipality including 19 workplaces with a population of 311 individuals. The municipality has implemented a model for human resource accounting, an extensive leader and co-worker development program and specific workplace based health and rehabilitation measures.

Methods: The studies compare co-workers and leaders self-ratings of health and psychosocial working conditions, and investigate how workplace health and rehabilitation programs affect these ratings by analysing quantitative results before, during and after carried out interventions. Qualitative data collection consisted of semi-structured interviews with middle managers and an examination of documents reviewing content of the interventions that had been carried out.

Findings: Results indicate differences concerning how the leaders and the co-workers judge their health and psychosocial working conditions. When comparing mean changes in scores on indexes by individuals grouped in the high quality workplace-based program group with individuals grouped in the low quality workplace-based program group, there were more positive mean changes for the former group.

Discussion: The findings of differences concerning how public sector leaders and co-workers judge their health and psychosocial working conditions give support for different job characteristics for these two categories of employees. The rather strong relationships in the assumed direction between employees' assessment of changes in health, and changes concerning stress and psychosocial working conditions that resulted are in line with other research studies. Comparing workplaces grouped as having high quality workplace-based programs with workplaces with low quality workplace-based programs indicates that workplace-based prevention and rehabilitation programs in public human service organizations using a broad change strategy with high levels of participation from both managers and co-workers, and developed leadership behaviour are more effective in improving employee health and psychosocial working conditions. 